\title{
Review of: Opposing roles for specific TIMPs in breast cancer prognosis
}

\author{
E. W. Thompson ${ }^{\mathrm{a}, \mathrm{b}}$, A. R. Mackay ${ }^{\mathrm{c}}$ \\ aSt Vincent's Institute of Medical Research, and ' University of Melbourne, Department of Surgery, \\ St Vincent's Hospital, Fitzroy, Victoria, 3065, Australia; 'Department of Experimental Medicine, \\ University of L'Aquila, Coppito 2, Via Vetoio, 67100 L'Aquila, Italy.
}

\section{Citation of the original article 1:}

Tumor tissue levels of tissue inhibitor of metalloproteinase-1 as a prognostic marker in primary breast cancer: A. S. Schrohl, M. N. Holten-Andersen, H. A. Peters, M. P. Look, M. E. Meijer-van Gelder, J. G. Klijn, N. Brunner, J. A. Foekens. Clinical Cancer Research 2004; 10(7): 2289-2298.

\section{Abstract of the original article 1}

Purpose: In the present study, we investigated the association between tumor tissue levels of tissue inhibitor of metalloproteinase-1 (TIMP-1) and prognosis in patients with primary breast cancer and analyzed whether TIMP-1 may be useful as a prognostic marker in combination with urokinase plasminogen activator (UPA) and plasminogen activator inhibitor type-1 (PAI-1).

Experimental design: In cytosolic extracts of 2984 primary breast tumors, total levels of TIMP-1 were determined using an established, validated ELISA. Levels of UPA and PAI-1 have previously been determined in the extracts.

Results: Univariate survival analysis showed a significant relationship between higher levels of TIMP-1 (continuous log-transformed variable) and poor prognosis (recurrence-free survival (RFS), overall survival (OS); $P<0.001$ ). Performing isotonic regression analysis, we identified a cut point to classify tumors as TIMP-1-low or TIMP-1-high. Using this cut point, high levels of TIMP-1 were significantly associated with shorter survival in univariate analysis, both in the total patient group (RFS, OS; $P<0.001$ ), in the node-negative subgroup (RFS, hazard ratio $=1.28$, $P=0.006$ ), and in the node-positive subgroup (RFS, hazard ratio $=1.43, P<0.001$ ). In multivariate analysis, including uPA and PAI-1, TIMP-1 was significantly associated with shorter RFS, both when included as a continuous log-transformed $(P=0.03)$ and as a dichotomized variable $(P=0.002)$.

Conclusions: This study validates previous findings that tumor tissue levels of TIMP-1 are associated with prognosis in patients with primary breast cancer. It confirms that TIMP-1 may be useful as a prognostic marker in combination with uPA/PAI-1 and adds substantial positive information on the use of TIMP-1 as a prognostic marker in breast cancer.

\section{Citation of the original article 2:}

Tissue inhibitors of metalloproteinase expression in human breast cancer: TIMP-3 is associated with adjuvant endocrine therapy success.

P. N. Span, R. L. Lindberg, P. Manders, V. C. Tjan-Heijnen, J. J. Heuvel, L. V. Beex, C. G. Sweep. J Pathol 2004; 202(4): 395-402.

Correspondence to: Erik W. Thompson, St Vincent's Institute of Medical Research, Fitzroy, Victoria, 3065, Australia. E-mail: rik@medstv.unimelb.edu.au; Tel: +6139288 2569; Fax: +61394160926 


\section{Abstract of the original article 2}

Tissue inhibitors of matrix metalloproteinase (TIMPs) may be involved in tumour growth, apoptosis, angiogenesis, invasion, and the development of metastases. This study has evaluated the association of the expression levels of the TIMP forms 1, 2, 3, and 4, measured by quantitative real-time RT-PCR, with classical clinicopathological characteristics, i.e. age, menopausal status, tumour size, histological grade, number of involved lymph nodes, and steroid hormone receptor status, and with disease progression and treatment sensitivity in 273 breast cancer patients. The mRNA levels of TIMP-1 and TIMP-2 were not associated with any known clinicopathological tumour feature. TIMP-3 and TIMP-4 levels were significantly higher in steroid hormone receptorpositive samples, although the levels of TIMP-4 were much lower than those of the other TIMPs. Only TIMP-3 predicted relapse-free survival (RFS) time differently depending on post-surgical treatment as, in particular, the interaction of TIMP-3 with endocrine therapy $(P=0.008, \mathrm{HR}=0.24,95 \% \mathrm{Cl}=0.09-0.69)$ contributed significantly to RFS in multivariate Cox regression analysis. In subgroup analyses, the 107 patients treated with tamoxifen differed greatly in prognosis after dichotomization by the median TIMP-3 level $(P=0.0003)$. Thus, high tumour levels of the matrix metalloproteinases inhibitor and pro-apoptotic factor TIMP-3 are associated with successful tamoxifen treatment of patients with breast cancer. Copyright 2004 Pathological Society of Great Britain and Ireland. Published by John Wiley \& Sons, Ltd.

\section{Review}

The two articles (Span et al. 2004 and Schrohl et al. 2004) highlight the complexity and specificity of matrix metalloproteinases (MMPs) and TIMPs in cancer biology, diagnosis, and treatment. Both provide novel insights into these issues by focussing on TIMPs rather than MMPs and extend a growing number of reports that the roles played by TIMPs in tumour pathology may be much broader than the inhibition of MMP action on the extracellular matrix $(E C M)$, and may extend to the regulation of MMP influence on many systems and potentially to MMPindependent functions. Specific TIMP-3 involvement in tamoxifen-induced apoptosis strongly contrasts the association between elevated TIMP-1 levels with poor outcome in breast cancer. Although candidate apoptotic mechanisms may elegantly explain the effect of TIMP-3 results, the reasons underlying the TIMP-1 result remain to be fully elucidated.

Tissue inhibitors of metalloproteinases (TIMPs) are endogenous inhibitors of the matrix metalloproteinase (MMP) subfamily (matrixins) of the zinc metalloproteinases [1]. The four TIMPs (TIMPs-1-4) characterized to date share significant homology, and a novel transmembrane MMP inhibitor RECK has been recently described [2]. Matrixins are neutral zinc endopeptidases which derive their name from their ability to collectively cleave all extracellular matrix (ECM) components (including collagens which are otherwise quite resistance to proteolysis).

Since malignant cancer cells degrade connective tissue structures, a paradigm has developed implicating MMPs (and other ECM proteases such as plasmin and cathepsins) in cancer growth, invasion, angiogenesis, and dissemination [3]. This in turn has led to development and clinical testing of a number of broad spectrum (e.g. BB2516/Marimastat British Biotech) and more selective (e.g. AG3340/Prinomastat, Agouron Phizer; BAY 12-9566/Tanomastat, Bayer) synthetic MMP inhibitors. These have generally failed to achieve sufficient positive outcomes for further development [4,5], highlighting the problems of targeting a system that to date comprises 26 MMPs exhibiting different substrate specificities. Indeed, in addition to ECM components, MMPs exhibit specific roles in the processing of an ever-expanding number of growth factors, cytokines and their cell surface receptors, angiogenic factors, and inhibitors. It is not surprising, therefore, that the targeting of specific MMPs within the tumour context may produce positive or negative results [6], making the overall effect of a broad-spectrum or even somewhat selective MMP inhibitor difficult to predict within the clinical setting. Furthermore, the high homology in the active sites may promote inhibitor activity against other MMP and related subfamilies such as ADAMs [6] which may in turn underlie the tendon toxicity which has compromised the dosing regimens in most if not all clinical trials. Finally without knowing the exact target MMP it is difficult to arrive at effective dosing regimens.

The determination of the MMP(s) critical for tumour maintenance and progression is critical for future therapeutic development. The papers described here demonstrate the benefit of measuring a specific component in an extensive archive and comparing the expression levels with clinical outcome. Other approaches include the laboratory testing of specific candidate MMPs and TIMPs, use of genetically engineered mice which lack specific candidates, and the development of more specific inhibitors such as 
the ADAM-sparing inhibitor BMS 275-291 by Bristol Myers Squibb, which unfortunately did not escape the connective tissue toxicity, and several other new MMP-selective inhibitors in development or clinical trial (e.g. Novartis, Pharmacia, Collagenex). Each of these is hoping to avoid toxicity by targeting important MMPs whilst sparing those which protect against tumour progression.

In the paper by Span [7], TIMP-3 associates with response to tamoxifen therapy in breast cancer illustrating a rare insight into the molecular mechanism through which the MMP/TIMP-3 axis enables tamoxifen to activate apoptosis. TIMPs-1-4 mRNA levels in 273 snap-frozen human breast cancers were compared to classical clinicopathological characteristics such as age, menopause status, tumour size, histological grade, lymph node involvement, and steroid hormone receptor (HR) status, as well as disease progression indices and treatment sensitivity. The authors found that TIMP- 3 and -4 levels were significantly higher in HR-positive samples, and that the presence of TIMP-3 specifically associated with better response to endocrine therapy. TIMP-3 has been shown to inhibit the growth, angiogenesis, invasion, and metastasis of experimental tumours [8-13], and has been directly implicated in apoptosis of normal and malignant cells $[9,10,14]$, in tumour necrosis factor alpha (TNF $\alpha$ ) receptor stabilization in colon carcinoma cells [15] and in Fas-induced cell death [16]. In contrast, TIMP-3 deficiency leads to accelerated apoptosis in the mouse mammary gland [17], and increased levels of TNF $\alpha$ in TIMP-3deficient mice leads to chronic hepatic inflammation and failure of liver regeneration due to hepatocyte apoptosis [18]. In relation to the study in review [7], it has been suggested that TIMP-3 may act as a mediator or sensitizer of p53-stimulated apoptosis driven by Fas: Fas ligand (FasL) ratios, which requires the MMP-inhibition capability of TIMP-3 $[16,19]$. However, complexity of MMP involvement in the regulation of Fas-mediated apoptosis is highlighted by the capacity of several MMPs (e.g. MMP$3,-7)$ which are inhibitable by all the TIMPs rather than TIMP-3 to specifically to degrade FasL. It is also degraded by certain ADAMs, but not ADAM-17, also known as TNF $\alpha$ convertase or TACE, the latter inhibited only by TIMP-3. Thus while the parallels can be drawn between the current study and previous observations relating Fas: FasL ratios to tamoxifen responsiveness they may be unrelated, and could be due to the production of soluble FasL receptor splice variants and/or direct tamoxifen regulation of FasL by oestrogen. The specificity of TIMP-3 for TACE inhibition and its specificity in predicting tamoxifen responsiveness certainly favour a role for TACE in the tamoxifen responsiveness. TNF $\alpha$ is a pleitrophic cytokine that can also serve as a potent tumour cell survival/growth factor [20] and thus could subvert the anti-proliferative effects of tamoxifen. Clearly coordinated research into this question in both laboratory and clinical arenas is required to better understand aspects of tamoxifen action that distinguish it from other chemotherapies, and permit the design of novel strategies to better predict tamoxifen responsiveness. An important practical consideration will be to determine whether TIMP-3 and apoptosis feature in aromatase inhibition of breast cancer cells. Aromatase inhibitors exhibit significant benefit in prevention trials, and are under consideration (and testing) for first-line adjuvant therapy against HR-positive breast cancer [21]. The potential exists for aromatase inhibitors to replace tamoxifen, and the pharmacogenomic potential of TIMP-3 to distinguish responsive patients would need to be assessed for aromatase. Validation of TIMP-3 protein levels and exploration of the mechanisms underlying loss of TIMP-3 from certain tumours will be an important next step (the TIMP-3 promoter is known to be methylated in various cancers) [22-25]. Ideally, TIMP-3 IHC analysis of the biopsy and/or ultimately a serum/plasma marker indicating TIMP-3 status could be developed and used to stratify patients likely to respond to tamoxifen.

Span et al. [7] also analysed TIMPs-1, -2, and -4 . TIMP-4 was also associated with oestrogen receptor (ER) status, but was dismissed because the levels were very low. Nonetheless, significant protein levels of TIMP-4 have been documented in breast cancer [26] and shown to either inhibit [27] or stimulate [28] experimental breast cancer. This is likely due to the different context of the models used, and the association here with ER suggests that further analysis in HR-positive models is warranted. The lack of association of TIMP-4 with any of the parameters may relate to the low mRNA levels, and analysis at the protein level could be more revealing and has important bearing on the issue of specificity/ mechanism as above. TIMP-1 did not associate with clinicopathological characteristics in the Span study [7]. This is surprising in view of the strong data reported by Schrohl et al. in the second paper under review [29], which validates their previous report [30] and other studies [31-34]. Not all breast cancer studies have shown a positive association with outcomeNakopoulou et al. [35] found reduced TIMP-1 in higher-grade breast cancers and an inverse association with proliferation. The different findings may relate to the methods employed. RNA and protein analysis in frozen tumour extracts may better indicate the causative TIMP-1 pool.

In any case both studies support the change in concepts concerning the roles of TIMPs in cancer 
biology in general, and breast cancer in particular [8]. This change is supported by:

- patterns of MMP expression do not always predict aggressive tumour behaviour in either human [36] or animal tumours [20];

- enhanced TIMP expression associates with poor outcome and tumour recurrence in a variety of cancers examined (reviewed in [37,38]);

- preoperative plasma levels of TIMP-1 have proved highly predictive of disease outcome in colon and rectal carcinoma $[39,40]$;

- MMPs

- degrade components of the plasmin-generating system downregulating cellular fibrinolytic activity,

- produce angiostatin from plasminogen-inhibiting angiogenesis,

- produce microplasminogen altering its macromolecular substrate specificity [41-45],

- under certain conditions may actually inhibit the invasive capacity of breast cancer cells $[45,46]$.

This may provide a basis for the association between increased tumour TIMP-1 levels and poor prognosis in breast cancer reported by annulling potential MMP-dependent protection against tumour progression/invasion.

Alternatively, it is possible that TIMP MMPinhibitory activity at the tumour site may be compromised providing a mechanism by which elevated tumour TIMP-1 expression could co-exist with MMPdependent tumour progression. In support of this, MMP-inhibitory activity of TIMP-1 and -2 , but not the activity of MMPs is exquisitely sensitive to inhibition by the thioredoxin redox system at physiologically attainable concentrations [36] and by $\mathrm{HOCl}$ at concentrations achievable at inflammatory loci [47]. Since the thioredoxin system and $\mathrm{HOCl}$ may make a significant contribution to breast cancer progression $[48,49]$, such a mechanism(s) for inhibition of TIMP activity could help explain its association with apparent disease progression.

In addition to its effects upon the MMP-mediated ECM degradation, TIMP-1 has been shown to stimulate cell proliferation in several cell types [50-52], which may require its anti-proteolytic activity. It also indirectly inhibits apoptosis [53] which would also impact upon tumour progression, and may have a nuclear function in breast cancer [54]. Thus, unlike the relatively neat assignment of TIMP-3 into apoptosis pathways required for tamoxifen responsiveness, the association of TIMP-1 with poor outcome remains unclear and could involve several mechanisms. These possibilities, and the observations reported in the study under comment, are supported by a recent report that overexpression of local tumour TIMP-1 does not suppress breast tumour growth in a transgenic mouse model but associates with increased proliferation [55].

The two papers reviewed here clearly show that like MMPs (as described above), TIMP expression cannot be regarded in a class-specific way, since despite general MMP inhibition each TIMP may have additional and independent roles. However, despite this potential complexity of TIMP function, they continue to hold promise as diagnostic and possibly therapeutic targets in breast cancer. Further definitive work along the lines of the excellent papers reviewed here, and the use of specific experimental models, will permit full understanding of TIMP involvement and its exploitation in breast cancer management.

\section{Acknowledgements}

The authors thank Dr. Barbara Fingleton, Vanderbilt, USA, for helpful discussions on TIMP-3 effects on apoptosis.

\section{References}

1. Woessner Jr JF. Matrix metalloproteinases and their inhibitors in connective tissue remodeling. FASEB 1991; 5: 2145-2154.

2. Baker AH, Edwards DR, Murphy G. Metalloproteinase inhibitors: biological actions and therapeutic opportunities. J Cell Sci 2002; 115: 3719-3727.

3. Stetler-Stevenson WG, Liotta LA, Kleiner DE. Extracellular matrix 6: role of matrix metalloproteinases in tumor invasion and metastasis. FASEB 1993; 7: 1434-1441.

4. Coussens LM, Fingleton B, Matrisian LM. Matrix metalloproteinase inhibitors and cancer: trials and tribulations. Science 2002; 295: 2387-2392.

5. Overall CM, Lopez-Otin C. Strategies for MMP inhibition in cancer: innovations for the post-trial era. Nat Rev Cancer 2002; 2: 657-672.

6. Egeblad M, Werb Z. New functions for the matrix metalloproteinases in cancer progression. Nat Rev Cancer 2002; 2: 161-174.

7. Span PN, Lindberg RL, Manders $P$ et al. Tissue inhibitors of metalloproteinase expression in human breast cancer: TIMP-3 is associated with adjuvant endocrine therapy success. J Pathol 2004; 202: 395-402.

8. Chambers AF, Matrisian LM. Changing views of the role of matrix metalloproteinases in metastasis. J Natl Cancer Inst 1997; 89: 1260-1270.

9. Ahonen M, Baker AH, Kahari VM. Adenovirus-mediated gene delivery of tissue inhibitor of metalloproteinases-3 inhibits invasion and induces apoptosis in melanoma cells. Cancer Res 1998; 58: 2310-2315.

10. Baker AH, George SJ, Zaltsman AB, et al. Inhibition of invasion and induction of apoptotic cell death of cancer cell lines by overexpression of TIMP-3. Br J Cancer 1999; 79: 1347-1355. 
11. Spurbeck WW, Ng CY, Strom TS, et al. Enforced expression of tissue inhibitor of matrix metalloproteinase-3 affects functional capillary morphogenesis and inhibits tumor growth in a murine tumor model. Blood 2002; 100: 3361-3368.

12. Collen A, Hanemaaijer R, Lupu F, et al. Membrane-type matrix metalloproteinase-mediated angiogenesis in a fibrin-collagen matrix. Blood 2003; 101: 1810-1817.

13. Qi JH, Ebrahem Q, Moore N, et al. A novel function for tissue inhibitor of metalloproteinases-3 (TIMP3): inhibition of angiogenesis by blockage of VEGF binding to VEGF receptor-2. Nat Med 2003; 9: 407-415.

14. Baker AH, Zaltsman AB, George SJ, Newby AC. Divergent effects of tissue inhibitor of metalloproteinase-1, -2 , or -3 overexpression on rat vascular smooth muscle cell invasion, proliferation, and death in vitro. TIMP-3 promotes apoptosis. J Clin Invest 1998; 101: 1478-1487.

15. Smith MR, Kung H, Durum SK, et al. TIMP-3 induces cell death by stabilizing TNF-alpha receptors on the surface of human colon carcinoma cells. Cytokine 1997; 9: 770-780.

16. Bond M, Murphy G, Bennett MR, et al. Tissue inhibitor of metalloproteinase-3 induces a Fas-associated death domain-dependent type II apoptotic pathway. J Biol Chem 2002; 277: 13787-13795.

17. Fata JE, Leco KJ, Voura EB, et al. Accelerated apoptosis in the TIMP-3-deficient mammary gland. J Clin Invest 2001; 108: 831-841.

18. Mohammed FF, Smookler DS, Taylor SE, et al. Abnormal TNF activity in TIMP3 - / - mice leads to chronic hepatic inflammation and failure of liver regeneration. Nat Genet 2004; 36: 969-977.

19. Edwards DR. TIMP-3 and endocrine therapy of breast cancer: an apoptosis connection emerges. J Pathol 2004; 202: 391-394.

20. Bisgaard HC, AR MacKay et al. "Spontaneous metastasis of rat liver epithelial cells transformed with $\mathrm{v}$-raf and v-raf/v-myc: association with different phenotypic properties." Invasion Metastasis 1997; 17(5): 240-50.

21. Grana G. Shifting paradigms in hormonal therapy for breast cancer. Cancer Biol Ther 2004; 3(9): 797-805.

22. Bachman KE, Herman JG, Corn PG, et al. Methylationassociated silencing of the tissue inhibitor of metalloproteinase-3 gene suggest a suppressor role in kidney, brain, and other human cancers. Cancer Res 1999; 59: 798-802.

23. Kang SH, Choi HH, Kim SG, et al. Transcriptional inactivation of the tissue inhibitor of metalloproteinase-3 gene by DNA hypermethylation of the $5^{\prime}-\mathrm{CpG}$ island in human gastric cancer cell lines. Int J Cancer 2000; 86: 632-635.

24. Ueki T, Toyota M, Sohn T, et al. Hypermethylation of multiple genes in pancreatic adenocarcinoma. Cancer Res 2000; 60: 1835-1839.

25. Wild A, Ramaswamy A, Langer P, et al. Frequent methylation-associated silencing of the tissue inhibitor of metalloproteinase-3 gene in pancreatic endocrine tumors. J Clin Endocrinol Metab 2003; 88: 1367-1373.

26. Zhao YG, Xiao AZ, Park HI, et al. Endometase/matrilysin-2 in human breast ductal carcinoma in situ and its inhibition by tissue inhibitors of metalloproteinases- 2 and -4 : a putative role in the initiation of breast cancer invasion. Cancer Res 2004; 64: 590-598.

27. Wang M, Liu YE, Greene J, et al. Inhibition of tumor growth and metastasis of human breast cancer cells transfected with tissue inhibitor of metalloproteinase 4 . Oncogene 1997; 14: 2767-2774.
28 Jiang $\mathrm{Y}$, Wang M, Celiker MY, et al. Stimulation of mammary tumorigenesis by systemic tissue inhibitor of matrix metalloproteinase 4 gene delivery. Cancer Res 2001; 61: 2365-2370.

29 Schrohl AS, Holten-Andersen MN, Peters HA, et al. Tumor tissue levels of tissue inhibitor of metalloproteinase-1 as a prognostic marker in primary breast cancer. Clin Cancer Res 2004; 10: 2289-2298.

30. Schrohl AS, Christensen IJ, Pedersen AN, et al. Tumor tissue concentrations of the proteinase inhibitors tissue inhibitor of metalloproteinases-1 (TIMP-1) and plasminogen activator inhibitor type 1 (PAl-1) are complementary in determining prognosis in primary breast cancer. $\mathrm{Mol}$ Cell Proteom 2003; 2: 164-172.

31. McCarthy K, Maguire T, McGreal G, et al. High levels of tissue inhibitor of metalloproteinase-1 predict poor outcome in patients with breast cancer. Int J Cancer 1999; 84: 44-48.

32. Visscher DW, Hoyhtya M, Ottosen SK, et al. Enhanced expression of tissue inhibitor of metalloproteinase-2 (TIMP-2) in the stroma of breast carcinomas correlates with tumor recurrence. Int $J$ Cancer 1994; 59: 339-344.

33. Ree AH, Florenes VA, Berg JP, et al. High levels of messenger RNAs for tissue inhibitors of metalloproteinases (TIMP-1 and TIMP-2) in primary breast carcinomas are associated with development of distant metastases. Clin Cancer Res 1997; 3: 1623-1628.

34. Nakopoulou L, Giannopoulou I, Stefanaki K, et al. Enhanced mRNA expression of tissue inhibitor of metalloproteinase-1 (TIMP-1) in breast carcinomas is correlated with adverse prognosis. J Pathol 2002; 197: 307-313.

35. Nakopoulou L, Giannopoulou I, Lazaris A, et al. The favorable prognostic impact of tissue inhibitor of matrix metalloproteinases-1 protein overexpression in breast cancer cells. APMIS 2003; 111: 1027-1036.

36. Farina AR, Tacconelli A, Cappabianca L, et al. Thioredoxin alters the matrix metalloproteinase/tissue inhibitors of metalloproteinase balance and stimulates human SK-N-SH neuroblastoma cell invasion. Eur J Biochem 2001; 268: 405-413.

37. Jiang Y, Goldberg ID, Shi YE. Complex roles of tissue inhibitors of metalloproteinases in cancer. Oncogene 2002; 21: 2245-2252.

38. Aljada IS, Ramnath N, Donohue K, et al. Upregulation of the tissue inhibitor of metalloproteinase- 1 protein is associated with progression of human non-small-cell lung cancer. J Clin Oncol 2004; 22: 3218-3229.

39. Holten-Andersen MN, Christensen IJ, Nielsen HJ, et al. Total levels of tissue inhibitor of metalloproteinases 1 in plasma yield high diagnostic sensitivity and specificity in patients with colon cancer. Clin Cancer Res 2002; 8: 156-164.

40. Holten-Andersen MN, Christensen IJ, Nilbert M, et al. Association between preoperative plasma levels of tissue inhibitor of metalloproteinases 1 and rectal cancer patient survival: a validation study. Eur J Cancer 2004; 40: 64-72.

41. Lijnen HR. Matrix metalloproteinases and cellular fibrinolytic activity. Biochemistry (Mosc) 2002; 67: 92-98.

42. Orgel D, Schroder W, Hecker-Kia A, et al. The cleavage of pro-urokinase type plasminogen activator by stromelysin-1. Clin Chem Lab Med 1998; 36: 697-702. 
43. Patterson BC, Sang QA. Angiostatin-converting enzyme activities of human matrilysin (MMP-7) and gelatinase B/ type IV collagenase (MMP-9). J Biol Chem 1997; 272: 28823-28825.

44. O'Reilly MS, Wiederschain D, Stetler-Stevenson WG, et al. Regulation of angiostatin production by matrix metalloproteinase-2 in a model of concomitant resistance. J Biol Chem 1999; 274: 29568-29571.

45. Farina AR, Tacconelli A, Cappabianca L, et al. Inhibition of human MDA-MB-231 breast cancer cell invasion by matrix metalloproteinase 3 involves degradation of plasminogen. Eur J Biochem 2002; 269: 4476-4483.

46. Deryugina El, Luo GX, Reisfeld RA, et al. Tumor cell invasion through matrigel is regulated by activated matrix metalloproteinase-2. Anticancer Res 1997; 17: 3201-3210.

47. Shabani F, McNeil J, Tippett L. The oxidative inactivation of tissue inhibitor of metalloproteinase-1 (TIMP-1) by hypochlorous acid $(\mathrm{HOCl})$ is suppressed by antirheumatic drugs. Free Radic Res 1998; 28: 115-123.

48. Haklar G, Sayin-Ozveri E, Yuksel M, et al. Different kinds of reactive oxygen and nitrogen species were detected in colon and breast tumors. Cancer Lett 2001; 165: 219-224.

49. Lincoln DT, Ali Emadi EM. Tonissen F, Clarke FM. The thioredoxin-thioredoxin reductase system: overexpression in human cancer. Anticancer Res 2003; 23: 2425-2433.
50. Gasson JC, Golde DW, Kaufman SE, et al. Molecular characterization and expression of the gene encoding human erythroid-potentiating activity. Nature 1985; 315: 768-771.

51. Luparello C, Avanzato G, Carella C, Pucci-Minafra I. Tissue inhibitor of metalloprotease (TIMP)-1 and proliferative behaviour of clonal breast cancer cells. Breast Cancer Res Treat 1999; 54: 235-244.

52. Yamashita K, Suzuki M, Iwata H, et al. Tyrosine phosphorylation is crucial for growth signaling by tissue inhibitors of metalloproteinases (TIMP-1 and TIMP-2). FASEB 1996; 396: 103-107.

53. Liu XW, Bernardo MM, Fridman R, Kim HR. Tissue inhibitor of metalloproteinase-1 protects human breast epithelial cells against intrinsic apoptotic cell death via the focal adhesion kinase/phosphatidylinositol 3-kinase and MAPK signaling pathway. J Biol Chem 2003; 278 : 40364-40372.

54. Ritter LM, Garfield SH, Thorgeirsson UP. Tissue inhibitor of metalloproteinases-1 (TIMP-1) binds to the cell surface and translocates to the nucleus of human MCF-7 breast carcinoma cells. Biochem Biophys Res Commun 1999; 257: 494-499.

55. Yamazaki M, Akahane T, Buck T et al. Long-term exposure to elevated levels of circulating TIMP-1 but not mammary TIMP-1 suppresses growth of mammary carcinomas in transgenic mice. Carcinogenesis 2004; 25: 1735-1746. 